\title{
Role of different nuclear charge radii parameterizations on the thermal equilibrium in nuclear reaction
}

\author{
Sangeeta $^{1}$ and Varinderjit Kaur $^{2, \text { a }}$ \\ ${ }^{1}$ School of Physics and Materials Science, Thapar University, Patiala - 147004 Punjab, India \\ 2 PG Department of Physics, Mata Gujri College, Fatehgarh sahib - 140406 Punjab, India
}

\begin{abstract}
We emphasize the role of nuclear charge radii parameterizations on the thermal equilibrium by studying the correlation between maximal value of average temperature achieved in highly interacting nuclear matter and nuclear stopping for mass symmetric and asymmetric reactions over the entire collision geometry within the framework of isospin-dependent quantum molecular dynamics (IQMD) model. Our study reveals that the increase in available phase space at initial stage through different nuclear charge radii parameterizations, enhance the temperature of nuclear system and reduces the nuclear stopping for both types of reactions. The influence of nuclear charge radii on the thermalization is more pronounced for mass symmetric reactions compared to mass asymmetric reactions. Moreover, the lighter colliding pair are good probe to study the role of nuclear radius in thermalization.
\end{abstract}

\section{Introduction}

The different reaction outcomes in mass symmetric and asymmetric nuclear reactions provides qualitative understanding of the different processes and nuclear dynamics involved [1,2]. A phenomenologist study of these reactions can provide the information about the energy dissipation and nuclear equation of state (NEOS) of asymmetric nuclear matter [3]. The mass asymmetry parameter is defined as: $\eta=\left|A_{T}-A_{P} / A_{T}+A_{P}\right|$, where $A_{T}$ and $A_{P}$ are mass number of target and projectile nuclei respectively. $\eta=0.0$ and $\eta \neq 0.0$ corresponds to mass symmetric and asymmetric nuclear reactions respectively. The study of these reactions (by keeping the total mass of the system same), actually conveys the structural effects where the total number of nucleons are equal with different combinations of the size of projectile and target nuclei [4]. From the literature, it has been concluded that the nuclear reaction dynamics is very much sensitive towards the structural effects. In the recent communications [5-8], the another way used to study the structural effects is via different nuclear charge radii parameterizations. Various observables of heavy ion collisions (HICs) have been studied at different reaction conditions to see the influence of nuclear charge radii parameterizations. The magnitude of transverse flow decreases and the energy of vanishing flow increases with increase in nuclear radius [5]. Also, the isospin-dependent and -independent forms of nuclear charge radii have been taken to spread light on the isospin as well as structural effects on multifragmentation, collective flow and nuclear stopping through nuclear radius [6-8].

The increment in radius affects the transverse as well as longitudinal momentum of nucleons from the initial state to the final fragment production stage of the reaction. The

a e-mail: drvarinderjit@gmail.com thermalization of the nuclear matter is completely related to the momentum of the nucleons. However, no such study exist in literature for mass asymmetric nuclear reactions. These declared facts have motivated us to emphasize the role of nuclear charge radii parameterizations on the thermal equilibrium in nuclear reactions (mass symmetric and asymmetric) at all geometry conditions. Though, there exist numerous of parameterizations of nuclear charge radii, we incorporated only four radii parameterizations i.e. $R_{L D M}$ [9], $R_{N G O}[10], R_{P P}[11,12]$ and $R_{R R}$ [13] so that, the calculated radius of a particular nucleus follow the trend: $R_{L D M}<R_{N G O}<R_{P P}<R_{R R}$. This kind of pattern have been selected to keep the study of structural effects via increase in nuclear radius in a systematic way. These nuclear charge radii parameterizations are briefly stated in Table 1. One can refer to Ref. [9-15] for the further details of these radii parameterizations.

The present work has been done by using Isospindependent Quantum Molecular Dynamics (IQMD) model $[16,17]$ and the temperature of the nuclear system is calculated by using the formalism: The Hot ThomasFermi approach $[18,19]$. Using the values of nuclear matter densities and kinetic energy densities of each particle at each time step, one can determine the values of temperature within the central sphere of $2 \mathrm{fm}$ radius.

The organization of the paper is as follow: Sect. 2 . explains the performed calculations and results. The concluding remarks are given in Sect. 3.

\section{Calculations and results}

The simulations have been carried out within the framework of IQMD model $[16,17]$ for the reactions of ${ }_{20}^{50} \mathrm{Ca}+{ }_{20}^{50} \mathrm{Ca}(\eta=0.0)$ and ${ }_{7}^{14} \mathrm{~N}+{ }_{36}^{86} \mathrm{Kr}(\eta=0.7)$ with fixed composite system mass number $A_{t o t}=100$ units and for the reactions of ${ }_{50}^{124} \mathrm{Sn}+{ }_{50}^{124} \mathrm{Sn}(\eta=0.0)$ and ${ }_{18}^{38} \mathrm{Ar}+{ }_{82}^{210} \mathrm{~Pb}$ $(\eta=0.7)$ with $A_{t o t}=248$ units over the entire range of

(c) The Authors, published by EDP Sciences. This is an Open Access article distributed under the terms of the Creative Commons Attribution License 4.0 (http://creativecommons.org/licenses/by/4.0/). 
Table 1. Different nuclear charge radii parameterizations: $R_{L D M}, R_{N G O}, R_{P P}$ and $R_{R R}$.

\begin{tabular}{|l|c|c|}
\hline Formula & Reference & Parameter \\
\hline$R_{L D M}=r_{0} A^{1 / 3}$ & {$[9]$} & $r_{o}=1.12 \mathrm{fm}$ \\
$R_{N G O}=\left(N r_{o_{n}} A^{1 / 3}+Z r_{o_{p}} A^{1 / 3}\right) / A$ & {$[10]$} & $r_{o_{n}}=1.1375+1.875 \times 10^{-4} A \mathrm{fm}$ \\
$R_{P P}=1.256 A^{1 / 3}(1-0.202 I)$ & {$[11,12]$} & $r_{o_{p}}=1.128 \mathrm{fm}$ \\
$R_{R R}=1.2332 A^{1 / 3}+\frac{2.8961}{A^{2 / 3}}-0.18688 A^{1 / 3} I$ & {$[13]$} & $I=(N-Z) / A$ \\
\hline
\end{tabular}

collision geometry at incident energy of $100 \mathrm{MeV} /$ nucleon. To observe the role of radius on thermalization, we examine the time evolution of the average scaled density $\left(<\rho^{a v g} / \rho_{o}>\right)$ and average temperature $\left(<T^{a v g}>\right)$ of the nuclear system as shown in upper and lower panels of Fig. 1 respectively, for the central mass symmetric $(\mathrm{Ca}+\mathrm{Ca})$ (left panels) and asymmetric $(\mathrm{N}+\mathrm{Kr})$ (right panels) reactions. Here, $\rho^{a v g}$ is the instantaneous density of all nucleons and $\rho_{o}$ is ground state density which is $0.17 \mathrm{fm}^{-3}$ in IQMD model. We have also done the same for the reactions of $\mathrm{Sn}+\mathrm{Sn}$ and $\mathrm{Ar}+\mathrm{Pb}$ (graphs not shown here). The conclusion is same as for the reactions of $\mathrm{Ca}+\mathrm{Ca}$ and $\mathrm{N}+\mathrm{Kr}$. The different lines in Fig. 1 corresponds to four different nuclear charge radii parameterizations as demonstrated in Table 1. For both types of reactions (mass symmetric as well as asymmetric), with increase in nuclear radius, the average scaled density of the nuclear system decreases apparently from initial level up to the time, $\mathrm{t} \approx 20 \mathrm{fm} / \mathrm{c}$. The role of radius on $<\rho^{a v g} / \rho_{o}>$ is maximum during the highly dense phase of the reaction when both the target and projectile nuclei completely overlap each other and diminishes as the system starts expanding. The increment in radius consequently enhance the the momentum of all nucleons and the average temperature of the system increases. This influence of radius on average temperature is maximum at $\mathrm{t} \approx 10$ $20 \mathrm{fm} / \mathrm{c}$ and reduces slowly as the system cools down. One can observe that the time at which the average scaled density and temperature attains its maximum value also increase with increase in radius which reveals that the nuclear system will take more time to saturate and cool down with large radius. This observation is also in agreement with Ref. [7].

The comparable role of radius on the average scaled density and temperature is different at different time for symmetric as well as asymmetric reactions. Therefore as a next step, we study the role of radius on thermalization by analyzing the maximal value of average temperature $\left(<T^{\text {avg }}>_{\text {max }}\right)$ of the highly complex nucleonic matter achieved during the time span of the nuclear reaction and nuclear stopping. The global parameter of nuclear stopping i.e. anisotropy ratio, is defined as:

$$
<R>=\frac{2}{\pi} \frac{\left[\sum_{i}^{A_{t o t}}\left|p_{\perp}(i)\right|\right]}{\left[\sum_{i}^{A_{t o t}}\left|p_{\|}(i)\right|\right]} .
$$

The transverse and longitudinal momentum of the $i^{\text {th }}$ particle are $p_{\perp}(i)=\sqrt{p_{x}^{2}(i)+p_{y}^{2}(i)}$ and $p_{\|}(i)=p_{z}(i)$ respectively. For complete nuclear stopping (or complete thermal equilibrium), the value of $\langle R\rangle$ is one. It is clear from the literature [20-23], that the maximal average temperature and nuclear stopping decreases with increase in scaled impact parameter i.e. $\hat{b}=\mathrm{b} / \mathrm{b}_{\max }$ where, $\mathrm{b}_{\max }=\left(R_{P}+R_{T}\right) f m,\left(R_{P}\right.$ and $R_{T}$ are radii of target and projectile nuclei respectively). Therefore, the present study also focused on the direct correlation

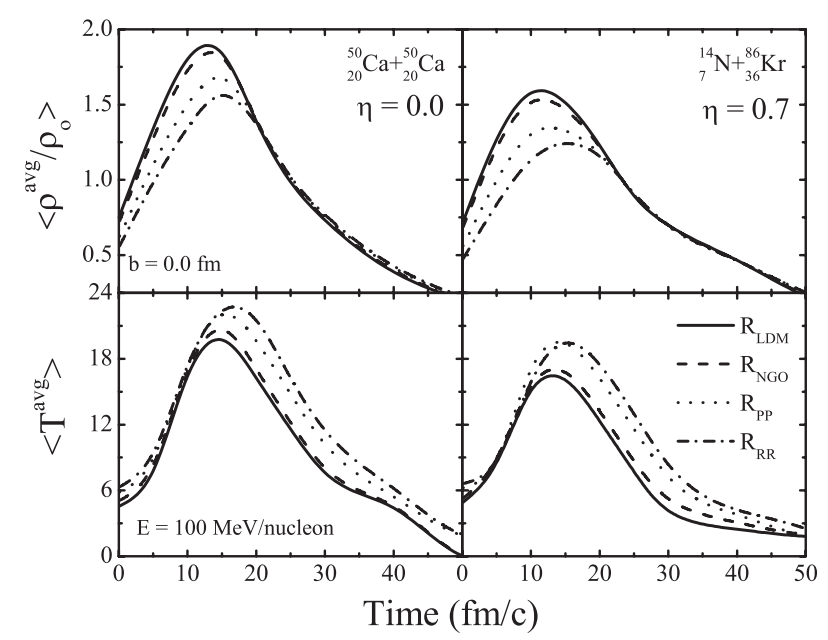

Figure 1. The time evolution of average scaled density (upper panels) and average temperature (lower panels) for central mass symmetric (left panels) and asymmetric (right panels) collisions with $A_{t o t}=100$ units at $\mathrm{E}=100 \mathrm{MeV} /$ nucleon for four different nuclear charge radii parameterizations (described by various lines).

between the temperature and the nuclear stopping of mass symmetric and asymmetric nuclear reaction. Figure 2 displays the maximal value of average temperature (achieved at $\mathrm{t} \approx 10-20 \mathrm{fm} / \mathrm{c}$ ) as a function of nuclear stopping at $\mathrm{t}=200 \mathrm{fm} / \mathrm{c}$ for mass symmetric (left panels) and asymmetric (right panels) nuclear reactions having $A_{\text {tot }}=100$ units (upper panels) and 248 units (lower panels) at $\mathrm{E}=100 \mathrm{MeV} /$ nucleon. The calculations have been done at six different collision geometries described by various symbols and for four different nuclear charge radii parameterizations described by various lines (i.e. $R_{L D M}, R_{N G O}, R_{P P}$ and $\left.R_{R R}\right)$ in the figure.

For mass symmetric reactions, the curves presenting the correlation, first increases with decrease in $\hat{b}$ and then shows saturation effect. This happen because, while going from semi-central to central collisions, the nuclear stopping still increases but the temperature of the system is not interestingly modified. Whereas, in mass asymmetric reactions, the curve shows straight line behavior, which means $<T^{a v g}>_{\text {max }}$ and $<R>$ increases monotonically with decrease in collision geometries. This is because, in mass symmetric reaction, the percentage participant matter (the nuclear matter which takes part in collision process) is almost same in central and nearly central collisions, whereas, the participant matter increases with decrease in $\hat{b}$ in mass asymmetric reactions.

It has been already observed by one of us that the nuclear stopping of mass symmetric reactions decreases with increase in radius [8]. This observation also holds true for the case of mass asymmetric reactions. The reason of reduction in nuclear stopping is that, with increase in radius the increment in longitudinal momentum is more compared to transverse momentum. Hence, there is 


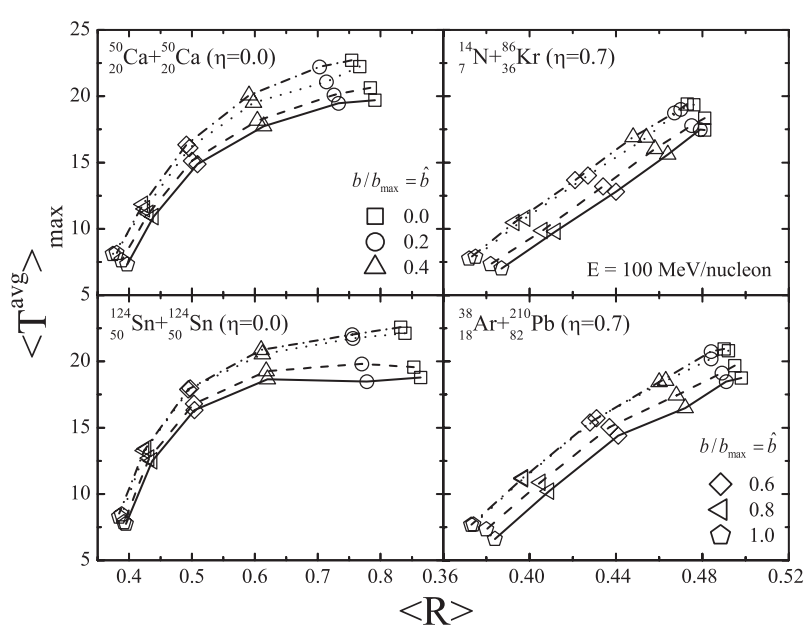

Figure 2. Correlation between maximal value of average temperature and nuclear stopping for mass symmetric (left panels) and asymmetric (right panels) nuclear reactions with $A_{\text {tot }}=100$ units (upper panels) and 248 units (lower panels) at $\mathrm{E}=100 \mathrm{MeV} /$ nucleon for six different collisions geometries (described by various symbols) and lines have same meaning as in Fig. 1.

less momentum transfer in transverse direction. Therefore enhanced longitudinal momentum (due to increase in radius) of the particles contributes more in raising the temperature of the system compared to transverse momentum. Because of these effects, the curves of correlation are lifted upward as we switch from $R_{L D M}$ to other three radii parameterizations. Obviously, the symmetric systems and the heavier systems are more thermalize compared to asymmetric systems and lighter systems respectively. We observe that the influence of radius is more pronounced in symmetric reactions and lighter reactions. The ratio of change in $\langle R\rangle$ as well as $\left\langle T^{a v g}>_{\max }\right.$ to the change in radius (while switching from $R_{L D M}$ to $R_{R R}$ ) is more for mass symmetric reactions (and also for lighter reactions i.e. with $A_{\text {tot }}=100$ units in present study) compared to mass asymmetric reactions (and also for heavier reactions i.e. $A_{t o t}=248$ units).

One can also see that for symmetric reactions, the role of nuclear charge radii parameterizations on the correlation of temperature and nuclear stopping decreases with increase in impact parameter; however, it remains almost same for asymmetric reactions through out the entire range of $\hat{b}$. This is because the lighter projectile and heavier target nucleus contributes differently in thermal equilibrium of mass asymmetric reactions at different collision geometries. These results also motivate to explore the role of nuclear charge radii parameterizations on the thermalization of mass asymmetric nuclear reaction explicitly for projectile and target nucleus for further studies.

\section{Summary}

In summary, we describe the influence of nuclear charge radii on the correlation between the temperature and the nuclear stopping. The study reveals that the change in nuclear radius at initial state $(\mathrm{t}=0 \mathrm{fm} / \mathrm{c})$ due to different nuclear charge radii parameterizations affects the maximal average temperature of the nuclear matter at $10-20 \mathrm{fm} / \mathrm{c}$ and the global nuclear stopping at $200 \mathrm{fm} / \mathrm{c}$. Change in phase space available to the nucleons at the initial level influences the reaction dynamics for the whole time span of highly interacting nuclear matter. The influence of nuclear charge radius is more prominent for mass symmetric as well as lighter reactions compared to mass asymmetric and heavier reactions respectively. Moreover, the role of nuclear charge radii on thermal equilibrium decreases with increase in $\hat{b}$ for mass symmetric reactions; however, remain almost equal for asymmetric reactions.

The financial support from the Department of Science and Technology (DST), Government of India in terms of INSPIREfellowship (grant No. DST/INSPIRE/03/2014/000234) and Young Scientist Award under the SERC Fast Track Scheme, wide letter no. SR/FTP/PS-020/2012 are gratefully acknowledged. We are also thankful to Prof. Rajeev K. Puri for giving access to his various computer programs for the current work.

\section{References}

[1] J. Singh, S. Kumar, R.K. Puri, Phys. Rev. C 62 , 044617 (2000)

[2] J. Singh, R.K. Puri, J. Phys. G: Nucl. and Part. Phys. 27, 2091 (2001)

[3] V. Kaur, S. Kumar, J. Phys. G: Nucl. and Part. Phys. 39, 085114 (2012)

[4] V. Kaur, S. Kumar, Phys. Rev. C 81, 064610 (2010)

[5] R. Bansal, S. Gautam, R.K. Puri, J. Aichelin, Phys. Rev. C 87, 061602 (2013)

[6] S. Gautam, Phys. Rev. C 88, 057603 (2013)

[7] Sangeeta, A. Jain, S. Kumar, Nucl. Phys. A 927, 220 (2014)

[8] Sangeeta, Acta Phys. Pol. B 47, 991 (2016)

[9] A. Bohr, B. Mottelson, Nuclear structure, vol. 1, W. A. Benjamin Inc., New York, Amsterdam (1969)

[10] H. Ngô, C. Ngô, Nucl. Phys. A 348, 140 (1980)

[11] B. Nerlo-Pomorska, K. Pomorski, Zeitschrift für Physik A Hadrons and Nuclei 344, 359 (1993)

[12] B. Nerlo-Pomorska, K. Pomorski, Zeitschrift für Physik A Hadrons and Nuclei 348, 169 (1994)

[13] G. Royer, R. Rousseau, The European Physical Journal A-Hadrons and Nuclei 42, 541 (2009)

[14] I. Dutt, R.K. Puri, Phys. Rev. C 81, 064608 (2010)

[15] I. Dutt, R.K. Puri, Phys. Rev. C 81, 064609 (2010)

[16] C. Hartnack, R.K. Puri, J. Aichelin, J. Konopka, S.A. Bass, H. Stöcker, W. Greiner, The European Physical Journal A-Hadrons and Nuclei 1, 151 (1998)

[17] C. Hartnack, H. Oeschler, Y. Leifels, E.L. Bratkovskaya, J. Aichelin, Physics Reports 510, 119 (2012)

[18] D.T. Khoa, N. Ohtsuka, M. Matin, A. Faessler, S. Huang, E. Lehmann, R.K. Puri, Nucl. Phys. A 548, 102 (1992)

[19] R.K. Puri, N. Ohtsuka, E. Lehmann, A. Faessler, M. Matin, D.T. Khoa, G. Batko, S. Huang, Nucl. Phys. A 575, 733 (1994)

[20] V. Kaur, S. Kumar, R.K. Puri, Nucl. Phys. A 861, 37 (2011)

[21] K. Singh Vinayak, S. Kumar, The European Physical Journal A-Hadrons and Nuclei 48, 1 (2012)

[22] A. Jain, S. Kumar, R.K. Puri, Phys. Rev. C 84, 057602 (2011)

[23] K.S. Vinayak, A.K. Chaudhuri, Journal of Physics G: Nuclear and Particle Physics 42, 025108 (2015) 